\title{
CENTRAL DENSITIES OF STAR CLUSTERS IN THE MAGELLANIC CLOUDS
}

\author{
Comparison with our Galaxy
}

\author{
M. KONTIZAS \\ Section of Astrophysics, Astronomy \& Mechanics, \\ Department of Physics, University of Athens \\ Panepistimiopolis GR-157 83, Athens, Greece \\ AND \\ D. GOULIERMIS AND E. KONTIZAS \\ Astronomical Institute, National Observatory of Athens, \\ P.O.BOX 20048, 11810 Athens, Greece
}

\begin{abstract}
The way star cluster systems in galaxies are forming and survive seem to depend on the relation of the central density $\rho$ (at half mass radius) of each cluster with its galactocentric distance $R_{g c}$. It is found that this relation takes the form of:

$$
\log \rho=A \times \log R_{g c}+B
$$

The cluster systems of our Galaxy and of the two Miagellanic Clouds, have been investigated. We have taken the cluster system of the conventional globulars of our Galaxy whereas the young and old systems of clusters in the LMC and SMC were treated separately. The radial distributions of central densities and half mass radii were found for all these systems showing a definite trend which depends on: $(\alpha)$ The total mass of the parent galaxy $\&(\beta)$ The age of the cluster system (young - old). It therefore appears that the total mass and/or the morphology of the parent galaxy plays a major role on the loci where clusters survive and form.
\end{abstract}

\section{Introduction}

The conventional definition of galactic globular clusters requires them to be dynamically old, populous stellar systems, relaxed under the two body realaxation mechanism. In the MCs very young populous globulars have been found, which didn't have the time to relax under the same mecha- 
nism. Therefore the Galactic globulars are first generation stellar systems, whereas the young globulars in the MCs are a second generation cluster population. We represent the central density $\rho$ of a globular cluster as the density of a spherical volume of radius $r_{h}$ where the half of the total mass of the cluster is contained [4]. This parameter - expressed in $M_{\odot} / p c^{3}$ - determines the total lifetime of a stellar system [11] and can provide a criterium for the separation between bound and unbound systems [8].

\section{Discussion}

Using previous catalogues ([5], [6], [7], [10], [1]) we estimated the half mass radii $r_{h}[12]$ and the central densities $\rho$ for the star clusters of LMC \& SMC with known dynamical parameters and we ploted these values versus the galactocentric distances $R_{g c}$ of the clusters. Then we created the same plots for the globular clusters of our Galaxy ([9], [3]). In all three galaxies for all cluster systems considered we found that the densities $\rho$ and the half mass radii $r_{h}$ are related to galactocentric distance $R_{g c}$ with trends of the form:

$$
\rho \propto R_{g c}{ }^{\gamma_{\rho}} \quad \text { and } \quad r_{h} \propto R_{g c}^{\gamma_{h}}
$$

where the slopes $\gamma$ are estimated by applying a best fit method.

The slopes $\gamma$ are varying for the various systems. More specifically for our Galaxy the slopes are steeper than those of the MCs with the old ones being steeper for the LMC clusters. There is also a difference in $\gamma \mathrm{s}$ between the young and old cluster systems in the MCs. The lower density systems are formed and seem to survive at larger galactocentric distances. It is also interesting to note that the maximum observed density values drop with the galaxy's total mass.

Finally we should note that the values of the slopes $\gamma_{\rho}$ and $\gamma_{h}$ we found for our Galaxy agree with those from references [2] and [13] respectively.

\section{References}

[1] Chrysovergis M., Kontizas M., Kontizas E.,1989, A. Ap. Sup. Ser., 77, 357

[2] Djorgovski S. \& Meylan G., 1994, A. J., 108(4), 1292

[3] Harris W.E., New Catalog of Globular Cluster Parameters, anonymous ftp to: physan.physics.mcmaster.ca

[4] King I., 1958, A. J., 63,110

[5] Kontizas M., 1984, A. Ap., 131, 58

[6] Kontizas M., Chrysovergis M., Kontizas E., 1987a, A. Ap. Sup. Ser., 68, 147

[7] Kontizas M., Hadjidimitriou D., Kontizas E., 1987b, A. Ap. Sup. Ser., 68, 493

[8] Lada, C. \& Lada, E. A., 1991, in Astr. Soc. Pas. Con. Ser., Janes K.(ed), 13, p1.

[9] Mandushev G., Spassova N., Staneva A., 1991, A. Ap. Sup. Ser., 252, 94

[10] Metaxa M., Kontizas M., Kontizas E., 1988, A. Ap. Sup. Ser., 73, 373

[11] Spitzer L. Jr., 1958, Ap. J., 127, 17

[12] Surdin V. G., 1979, Sov. Astron., 23(6), 648

[13] Van den Bergh S., 1994, A. J., 108(6), 2145 